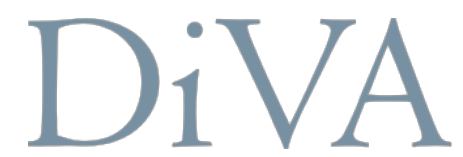

http://uu.diva-portal.org

This is an author produced version of a paper published in Journal of Solid State Electrochemistry. This paper has been peer-reviewed but does not include the final publisher proof-corrections or journal pagination.

Citation for the published paper:

Agmo Hernández, Victor

"The theory of metal electronucleation applied to the study of fundamental properties of liposomes"

Journal of Solid State Electrochemistry, 2013, 17,2 (SI): pp 299-305

URL: http://dx.doi.org/10.1007/s10008-012-1874-5

Access to the published version may require subscription. 


\title{
The theory of metal electronucleation applied to the study of fundamental properties of liposomes.
}

\author{
Víctor Agmo Hernández \\ Department of Chemistry-BMC, Uppsala University. Husargatan 3, Box 579, \\ 75123, Uppsala, Sweden \\ Phone: +46184713635 \\ Fax: +46184713654 \\ E-mail: victor.agmo@kemi.uu.se
}

\begin{abstract}
This short review describes how the theory of electrochemical metal nucleation considering nonstationary effects due to the activation of latent nucleation sites has been successfully translated and applied to describe phenomena observed on lipid membranes. This rather unexpected connection is merely formal, but has resulted in a completely new approach in liposome research. It has been proposed that hydrophobic active sites spontaneously and constantly appear and disappear on lipid membranes. These sites control the affinity of liposomes for hydrophobic surfaces and determine the permeability of the lipid membrane to small hydrophilic molecules. Thus, the kinetic models for liposome adhesion on hydrophobic substrates and for the spontaneous leakage of liposomal content are identical to that of non-stationary nucleation mentioned above. Therefore, the broad scope of the available work on metal nucleation has facilitated the interpretation of the data obtained in liposome research. Future applications of the nucleation model in the realm of liposomes are also discussed.
\end{abstract}

Keywords: Liposomes, nucleation kinetics, hydrophobic active sites, mercury electrode

The author dedicates this contribution to Prof. Alexander Milchev on his $70^{\text {th }}$ birthday. I had the honour of meeting Prof. Milchev in Sofia in 2008, and his kindness and wisdom had a profound impact on me. His encouraging words were, and still are, a great source of inspiration. 


\section{Introduction}

Lipid vesicles or liposomes are spherical structures consisting of a lipid bilayer membrane separating an internal aqueous compartment from the exterior aqueous environment (Fig. 1). They are formed spontaneously when certain amphiphilic lipids (such as lecithins) are suspended in water. They are commonly used as models of biological cell membranes, as the core of the latter is also a lipid bilayer. Studies on cell morphology and on the general properties of cell membranes can be performed using liposomes, which can be prepared in such a way as to mimic the composition of biological membranes. Membrane proteins can be included into the liposomal membrane, and their function be studied under well controlled conditions. Liposomes are also useful as drug delivery systems, as they may contain therapeutic agents either in their aqueous interior or embedded in the lipophilic membrane. Examples of these and other applications of liposomes have been reviewed previously [1-6].

In spite of the great interest in liposomes from both the industrial and scientific points of view, several of their properties are still not completely understood and are a constant matter of debate among researchers. For example, it is well known that the inclusion of cholesterol in phospholipid membranes condenses the lipid packing, increases the stability of the liposome and, at the same time, maintains the membrane fluid even if the temperature drops below the main phase transition. However, there is still no consensus on the mechanisms that lead to the tighter packing of the lipids and the aforementioned effects [7]. The interaction of liposomes with hydrophilic and hydrophobic substrates is also a matter of current discussion. On the former, some researchers claim that single liposomes can attach and rupture on the surface of the substrate, forming a supported lipid bilayer (SLB) if the substrate is smooth enough [8,9]. Other researchers, however, have found that intact liposomes first accumulate on the surface and just rupture after a critical surface concentration has been achieved [10,11]. Mixed cases have also been reported, with some liposomes from a sample rupturing and spreading instantly while others accumulate first on the surface [12]. In other words, a general consensus is still lacking. The situation is even more complex when referring to the adhesion, rupture and spreading of liposomes on hydrophobic substrates, where the formation of supported lipid bilayers [13-15], supported 
lipid monolayers [16-18], and immobilized liposome layers [10,19] has been reported. The level of hydrophobicity and smoothness of the surface being studied have been proposed as possible reasons behind these discrepancies [13]. The actual mechanism by which the liposomes rupture on the hydrophobic substrate is also a matter of discussion, although convincing models have been recently proposed [20-22]. Why liposomes in suspension adhere at all to hydrophobic substrates given that their surface is mainly hydrophilic is another subject of speculation. A convincing explanation, as unlikely as it may seem at first sight, was first inspired by the kinetic model for non-stationary metal electronucleation at active sites on electrodes described by Milchev [23]. This mini-review will focus on this approach, represented by the "liposome nucleation model". This model has served to explain some observable properties of liposomes and liposome suspensions, resulting in a whole new approach in liposome research. The first part of the review briefly describes the electrochemical experiments that provided with the observations that first suggested the analogy between liposome adhesion and metal nucleation. Secondly, the model itself and how it served to explain the adhesion of liposomes on mercury electrodes is presented. Finally, the use of the model to explain observed properties of liposomes in suspension and to account for discrepancies in the literature is discussed.

\section{The adhesion-spreading of liposomes on mercury electrodes}

As has been reported previously [16,17,21], the adhesion, rupture and spreading of multilamellar and giant unilamellar vesicles (MLVs and GUVs, respectively) on mercury electrodes can be characterized with chronoamperometric measurements. As liposomes rupture and spread on mercury electrodes forming a supported lipid monolayer [18], the rupture of single liposomes produces adsorbed lecithin islands on the mercury surface. The surface charge density and capacitance on these islands is different than that of the bare mercury surface. Therefore, the formation of the individual islands is coupled with the flow of capacitive currents, which are recorded as spikes in chronoamperometric measurements $[16,17]$ as shown in Fig. 2a. Individual adhesion and spreading events can therefore be identified (shown in Fig. 2b). Furthermore, the mathematical analysis of the charge transients obtained after integration of a 
single spike allows determining the "micro-kinetics" of the adhesion-spreading process, i.e. the kinetics of the adhesion and spreading of a single liposome. In turn, the determined quantitative micro-kinetic parameters (time constants and activation energies) are related to the membrane fluidity, elasticity and resistance to rupture, as has been shown in several publications [17,21,24]. Furthermore, by counting the number of adhesion-spreading events occuring on a certain time period, one can get information concerning the "macro-kinetics" which is related to the interaction of the liposome suspension and the mercury surface as a whole. This interaction is also related to the properties of the lipid membrane. Analysis of macro-kinetic data (number of adhesion spreading events over time per surface area and how it changes with temperature) has been proven useful to perform qualitative studies of real cell membranes, such as thrombocytes and mitochondria, as well as to study the effect of foreign molecules and the liposome composition on the membrane properties [25-28].

When several repetitions of the chronoamperometric experiments are performed, one can plot the time dependence of the average number of signals recorded $(\mathrm{N}(t)$ vs. $t$ plot, where $\mathrm{N}$ is the number of events recorded during a time $t$ after formation of the mercury drop). The obtained curve deviates significantly from what would be expected both from a pure diffusion- (Fig. 3) and from a pure kinetically- controlled process, suggesting a mixed control of the overall reaction. Furthermore, temperature dependence experiments showed that increasing the temperature decreased the number of recorded adhesion-spreading events during a certain time, suggesting that a reversible reaction should be involved. The first proposed solution was that the kinetics of adsorption played a role in determining the rate of the overall reaction [17]. However, it was shown that decreasing the surface charge density on the mercury decreased the rate at which events took place (even when correcting for the loss of sensitivity expected) [29], an effect contrary to what would be expected fron an adsorption-controlled process. 


\section{The "nucleation model": Latent active sites in liposomes}

The solution to this problem was first elucidated in collaboration with Milchev [29] and was based in the following assumptions: i) liposomes initially have no affinity for the hydrophobic mercury surface. ii) when the liposomes are close to the mercury they can be reversibly "activated", gaining affinity for the surface and iii) only these active liposomes will undergo adhesion-spreading and produce a signal. In other words, the kinetic part of the overall adhesion-spreading reaction is given by:

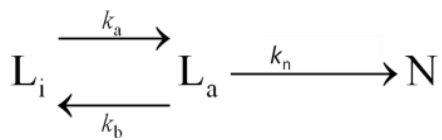

\section{(Scheme I)}

where $\mathrm{L}_{\mathrm{i}}$ is an inactive liposome, $\mathrm{L}_{\mathrm{a}}$ is an active liposome and $\mathrm{N}$ is a liposome attached to the surface (a nucleus), which will fastly rupture and spread on the surface, $k_{\mathrm{a}}$ and $k_{\mathrm{b}}$ represent, respectively, the rate constants of formation and consumption of active sites in the lipid membrane (activation of the liposome) and $k_{\mathrm{n}}$ is the rate constant for the actual attachment or "docking" of the liposome on the surface. Formally, Scheme I is identical to the mechanism for electrochemical nucleation after activation of latent active sites described by Milchev [23,30,31], and is therefore referred to as the "nucleation model".

A general solution for the number of observed adhesion-spreading events is obtained by combining the kinetic equations derived from Scheme I with the equations decribing the mass transport from the bulk to the mercury surface. The obtained expression is given by [29]:

$$
\mathrm{N}(t)=A_{\mathrm{SMDE}} C_{\mathrm{lip}} \sqrt{D} A_{1} \operatorname{erf} \sqrt{-k_{\mathrm{o} 1} t} e^{-k_{\mathrm{o} 1} t}+A_{2} \operatorname{erf} \sqrt{-k_{\mathrm{o} 2} t} e^{-k_{\mathrm{o} 2} t}+A_{3} \sqrt{t}
$$

where $A_{\mathrm{SMDE}}$ is the surface area of the mercury electrode, $C_{\text {lip }}$ is the bulk concentration of liposomes, $D$ is the diffusion coefficient of the liposomes, erf is the error function and $A_{1}, A_{2}, A_{3}, k_{\mathrm{o} 1}$ and $k_{\mathrm{o} 2}$ are parameters derived from the activation, deactivation, and docking/nucleation rate constants. Eq. 1 successfully predicts the average number of adhesion-spreading events obtained after a certain time, as shown in Fig. 3. Further extrapolating the vast knowledge on metal 
nucleation to the realm of liposome adhesion and spreading on mercury, several features of the latter were discovered. For example, it was found that the temporal distribution of the recorded adhesion-spreading events follow the same trend than that of the formation of metal nuclei on electrodes. Both processes are stochastic in nature and the distribution of the number of events recorded at a certain time is described by the Poisson distribution (Fig. 4) [32]. From the chronoamperometric experiments, it was first proposed that the random appearance of inverted lipid molecules (with their lipophilic tail pointing towards the aqueous environment) was the origin of the stochastic nature of the nucleation-like features of liposome adhesion and spreading on mercury electrodes. These inverted lipid molecules would form the "active sites" described in the nucleation model. As will be discussed in following sections, the proposed active sites in liposomes affect important fundamental properties of lipid membranes, and may help to explain contradictory data found in the literature.

\section{Non-electrochemical studies: Activation- deactivation as an intrinsic, fundamental process in lipid membranes.}

\subsection{Adhesion and spreading of liposomes on hydrophobic nanoparticles.}

The fact that, according to the electrochemical experiments described above, the adhesion-spreading of liposomes on mercury was enhanced when the charge surface density on the electrode was relatively high, suggested that the electric field acting on the lipid membrane was responsible for the formation of active sites on the liposomes [29]. However, adhesion-spreading of liposomes on mercury surfaces has been recorded also at the point of zero charge [33]. According to the nucleation model, this observation imply that the activation of liposomes may occur even if no electric fields are present. In order to test this hypothesis, Agmo Hernández et al. [34] studied the interactions between hydrophobic nanoparticles and liposomes in different phase states. In short, large unilamellar vesicles (LUVs, $100 \mathrm{~nm}$ diameter) formed either by dipalmitoyl phosphatidyl choline (DPPC) or by palmitoyl oleoyl phosphatidylcholine (POPC) 
were mixed with a large excess of polystyrene nanoparticles (115 $\mathrm{nm}$ diameter) stabilized by sulfate anions. All experiments were performed at $25{ }^{\circ} \mathrm{C}$. At this temperature DPPC liposomes are in the gel phase state, while POPC liposomes are found in the liquid crystalline phase state. The media in which the liposomes and the particles were suspended was rich in ions $(10 \mathrm{mM}$ phosphate and $150 \mathrm{mM}$ $\mathrm{NaCl}, \mathrm{pH}=7.4$ ) in order to decrease all electrostatic interactions and to mimic physiological conditions. The liposomes were filled with an isotonic solution containing $100 \mathrm{mM}$ of the fluorescent molecule carboxyfluorescein (CF). At this concentration, the fluorophore self-quenches, giving almost no fluorescence response. Upon rupture and spreading of the liposomes on the surface of the nanoparticles, the fluorophore is released and diluted in the bulk solution, giving a clear fluorescence response. The rate at which the fluorescence of the liposomeparticles mixture increases is therefore an indication of the rate at which liposomes fuse with the particles. These experiments were complemented with cryo-transmission electron microscopy (Cryo-TEM), which allows observing the adhesion-spreading reaction at different stages; and with dynamic light scattering (DLS), which allowed following any changes in the size distribution of the samples. Kinetic analysis of the fluorescence experiments performed with DPPC liposomes in the gel phase state agreed with the following reaction model:

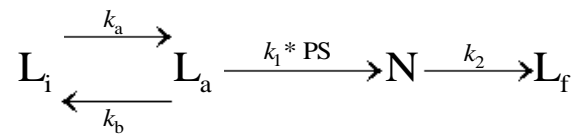

(Scheme II)

which is in principle the same reaction mechanism as the one shown in Scheme I. The difference is the inclusion of an additional step (going from $\mathrm{N}$ to $\mathrm{L}_{\mathrm{f}}$ ) which represents the transition from an adhered ("nucleated") liposome (N) to an spread lipid monolayer $\left(\mathrm{L}_{\mathrm{f}}\right)$ (Fig. 5). In the case of the adhesion and spreading on mercury (Scheme I), this last step is much faster than the preceeding ones, and is therefore practically simultaneous to the nucleation step. However, in the case of adhesion and spreading on nanoparticles, DLS and Cryo-TEM clearly demonstrated that $\mathrm{N}$ has a very long lifetime, in the span of a few hours, and its transformation into a supported lipid monolayer should therefore be considered in the kinetic analysis. Remarkably, when the experiment was performed with POPC liposomes in the liquid crystalline phase state, very large (sometimes visible to the naked eye) particle-liposome clusters appeared and the release of CF was 
prevented, more likely because intact liposomes were captured within large particle clusters (Fig. 6). This was explained considering that liquid crystalline liposomes may present more than one active site at once, due to the highly dynamic nature of their lipid membrane. Therefore, the attachment to more than one particle is likely to occur, thus preventing rupture and spreading.

As shown above, the fusion of DPPC liposomes with polystyrene nanoparticles is formally preceeded by an activation-deactivation process equivalent to the one observed for adhesion-spreading in mercury. However, the determined time constants for the two different cases differ by several orders of magnitude: a few microseconds in the case of charged mercury electrodes and several hours in the case of hydrophobic nanoparticles. It is necessary to consider that, on mercury, only the successful activation-nucleation processes are taken into account (single events). On the other hand, the fluorescence measurements consider as well all the formed active sites that did not cause any nucleation and were subsequently deactivated (the behaviour of the whole suspension). In other words, the mercury experiments measure the time constant of the formation of a single active site, regardless of the rate at which this site can be reversibly deactivated. The fluorescense experiments measure the time constant for the increase in the number of active sites in the whole suspension, which is related with the rate of deactivation of these sites in the microscopic level.

In conclusion, it was shown that liposomes need to undergo an activation process before attaching to an hydrophobic surface. This activation occurs even when no electric fields are present, although a charged surface may catalize their formation. The remaining question is if the activation process may occur spontaneously even if no foreign substrate is available. Studying the spontaneous leakage of CF from LUVs shed some light on this topic, as is shown below.

\subsection{Spontaneous leakage of CF from LUVs.}

The spontaneous leakage of entrapped molecules is a topic of major interest, especially in the design of liposomes for drug delivery applications. Most liposomes applied in this field are required to encapsulate a compound for rather long periods of time and to release it only when necessary. It is therefore of the utmost importance to determine the rate at which a certain compound leaks from a liposome. Although determining how much of a compound has leaked can be a 
simple task, contradictory data is often found in the literature, with the same liposomes showing different leakage rates for the same compound under the same conditions (examples, references [35-37]).

Without the addition of the polymer nanoparticles, fluorescence experiments similar to the ones described in the previous section allow measuring the rate at which the fluorophore $(\mathrm{CF})$ spontaneously leaks from the liposomes. The report by Agmo Hernández et al. cited above [34], demonstrated that the long-term (over 10 hours) leakage profile of CF from both DPPC and POPC liposomes cannot be described by a single step process. The data could only be fitted to a theoretical model if two sequential or parallel processes were considered. Even more interestingly, one of those processes should involve only the liposomes and not their contents. In other words, the rate of spontaneous leakage of $\mathrm{CF}$ is dependent not only on the transfer of CF from one side of the membrane to the other, but also on intrinsic changes occurring in the lipid membrane. The approach that best fitted the obtained data corresponds to the notion of the nucleation model, and is given by the reaction mechanism:

$$
\mathrm{CF}_{\text {response }} \stackrel{k_{\mathrm{L} 1}}{\longleftarrow} \mathrm{CF}\left(\mathrm{L}_{\mathrm{i}}\right) \stackrel{k_{\mathrm{a}}}{\longleftarrow} \mathrm{CF}\left(\mathrm{L}_{\mathrm{a}}\right) \stackrel{k_{\mathrm{L} 2}}{\longrightarrow} \mathrm{CF}_{\text {response }}
$$

where $\mathrm{CF}\left(\mathrm{L}_{\mathrm{i}}\right)$ and $\mathrm{CF}\left(\mathrm{L}_{\mathrm{a}}\right)$ represents the $\mathrm{CF}$ trapped in inactive and active liposomes, respectively. $k_{\mathrm{L} 1}$ and $k_{\mathrm{L} 2}$ are the rate constants for the spontaneous leakage of $\mathrm{CF}$ from the two different kinds of liposomes and $k_{\mathrm{a}}$ and $k_{\mathrm{b}}$ are equivalent to those described in Scheme II. The solution of the kinetic equations describing Scheme III is, evidently, identical to the solution provided by Milchev for the nucleation of metals:

$$
x_{\mathrm{CF}_{\text {Released }}} t=1+\left(\frac{A^{\prime}-\lambda^{\prime}-P}{2 \lambda^{\prime}}\right) e^{-\frac{A^{\prime}+\lambda^{\prime}}{2} t}-\left(\frac{A^{\prime}+\lambda^{\prime}-P}{2 \lambda^{\prime}}\right) e^{-\frac{A^{\prime}-\lambda^{\prime}}{2} t}
$$

where

$$
A^{\prime}=k_{\mathrm{a}}+k_{\mathrm{b}}+k_{\mathrm{L} 1}+k_{\mathrm{L} 2}, \quad \lambda^{2}=k_{\mathrm{a}}-k_{\mathrm{b}}+k_{\mathrm{L} 1}-k_{\mathrm{L} 2}{ }^{2}+4 k_{\mathrm{a}} k_{\mathrm{b}}
$$
and $P=2 k_{\mathrm{L}_{1}} x_{\mathrm{CF}_{\mathrm{L}_{\mathrm{i}}}}^{0}+k_{\mathrm{L}_{2}} x_{\mathrm{CF}_{\mathrm{L}_{\mathrm{a}}}}^{0}$ and $x_{\mathrm{CF}_{\mathrm{L}_{\mathrm{a}}}}^{0}$ and $x_{\mathrm{CF}_{\mathrm{L}_{\mathrm{i}}}}^{0}$ are the initial fractions of total $\mathrm{CF}$ inside the active and the inactive liposomes respectively. This is obviously a direct translation of the knowledge on metal nucleation to the realm of liposomes. 
Experiments with liposomes of different ages (measured from the time at which the preparation was ready) showed that freshly prepared liposomes leaked much faster than liposomes which had been incubated for 24 hours, even though the time constants describing the observations remained basically unchanged. The changes were reflected in the initial conditions of the experiment, suggesting that the relative proportions of $\mathrm{L}_{\mathrm{i}}$ and $\mathrm{L}_{\mathrm{a}}$ change overtime until the equilibrium is reached. It was also observed that liposome suspensions that leaked faster also reacted more readily with polystyrene nanoparticles, supporting thus the assumption that the same activation-deactivation process is involved in both processes. The values for $k_{\mathrm{L} 1}$ for DPPC and POPC liposomes were very close to zero, implying that most of the leakage of the CF occurs through the active sites. Discrepancies in the literature as those mentioned above (references [35-37]) could have arisen because the age of the liposomes employed was not considered. Usually, this is a parameter not controlled or omitted in most reports. The findings by Agmo Hernández et al. have shown that it may play a very important role in determining the rates of spontaneous leakage of hydrophilic compounds [34].

The fact that the active sites provide with affinity for hydrophobic surfaces and are responsible for the permeability towards water soluble molecules, suggest that these sites may be formed by hydrophobic patches on the membrane surface. In agreement with what was proposed for the active sites formed prior to the adhesion and spreading on mercury electrodes, it is likely that lipid molecules partially turned around may constitute the core of the active site. Therefore, the active sites have also been termed "hydrophobic defects" or "hydrophobic active sites". Recent molecular dynamic simulations have in fact shown that, at a certain given time, a large proportion of the surface area of a liposome is actually hydrophobic $[38,39]$. One can speculate that the short-lived hydrophobic patches described in these publications may in some cases join with others, growing in size and increasing their stability and life-span, resulting in an hydrophobic active site as the ones suggested by the experiments by Agmo Hernández et al.

\section{General conclusions and future directions}

The most interesting conclusion obtained from this series of experiments is that undisturbed liposomes in suspension form a heterogeneous mixture of active and inactive vesicles. Furthermore, the two populations are not independent, but are 
found in dynamic equilibrium, meaning that it is virtually imposible to get a longlived homogeneous liposome suspension. However, one can in principle control the conditions in order to shift the equilibrium towards the kind of suspension that is desired.

Current investigations have shown that other properties of lipid membranes, as well as other processes occurring in liposome suspensions, may involve or be affected by the proposed activation-deactivation of the membrane. It has been observed, e.g., that the tendency of liposomes to rupture on hydrophilic substrates is directly related to their degree of activation (unpublished data). This could explain why different adhesion-spreading mechanisms have been observed for liposomes on hydrophilic surfaces [10-12]. Active liposomes may rupture and spread instantly, while inactive liposomes may need to accumulate first on the surface, explaining the discrepancies reported. The role of hydrophobic active sites in the process of liposome-liposome fusion is also a matter of study. In principle, the formation of hydrophobic defects in two liposomes fusing will facilitate their initial contact, as the repulsive forces arising from the hydration layer would be diminished. Preliminary, as yet unpublished, results agree with the notion of an activation of the liposome membrane preceeding liposome-liposome fusion.

The idea of translating the knowledge of metal nucleation to the study of liposome membranes has resulted in a completely new research direction. In the short period of time elapsed since the analogies between the two systems were first pointed out [32,29], the application of the nucleation model to study the behavior of liposomes has provided with new insights into the dynamics at molecular level of these interesting structures. Even though the actual formation of active sites remains to be demonstrated, the evidence gathered so far has convincingly show that the model can be used to describe the experimental observations accurately. This new area of study is the center of several on-going projects and, as the knowledge and challenges increase, the involvement of more researchers is expected. The author hopes that, in the future, this approach will help to unravel some more unique properties of lipid membranes. 


\section{Acknowledgments}

The author thanks Prof. Katarina Edwards, Department of Chemistry-BMC, Uppsala University for fruitful and interesting discussions on the subject and for providing the resources and facilities for the completion of this work.

\section{References}

1. Lasic DD (1998) Trends Biotechnol 16:307-321

2. Mishra GP, Bagui M, Tamboli V, Mitra AK (2011) Recent applications of liposomes in ophtalmic drug delivery. J Drug Deliv. Doi: 10.1155/2011/863734

3. Fielding RM, Lasic DD (1999) Expert Opin Ther Pat 9:1679-1688

4. Lasic DD, Papahadjopoulos D (1996) Curr Opin Solid St M 1:392-400

5. Lasic DD, Templeton NS (1996) Adv Drug Deliver Rev 20:221-266

6. Templeton NS, Lasic DD (1999) Mol Biotechnol 11:175-180

7. Daly TA, Wang M, Regen SL (2011) Langmuir 27:2159-2161

8. Radler J, Strey H, Sackmann E (1995) Langmuir 11:4539-4548

9. Williams LM, Evans SD, Flynn TM, Marsh A, Knowles PF, Bushby RJ, Boden N (1997)

Langmuir 13:751-757

10. Keller CA, Kasemo B (1998) Biophys J 75:1397-1402

11. Keller CA, Glasmastar K, Zhdanov VP, Kasemo B (2000) Phys Rev Lett 84:5443-5446

12. Johnson JM, Ha T, Chu S, Boxer SG (2002) Biophys J 83:3371-3379

13. Lipkowski J (2010) Phys Chem Chem Phys 12:13874-13887

14. Xu S, Szymanski G, Lipkowski J (2004) J Am Chem Soc 126:12276-12277

15. Sek S, Xu S, Chen M, Szymanski G, Lipkowski J (2008) J Am Chem Soc 130:5736-5743

16. Hellberg D, Scholz F, Schauer F, Weitschies W (2002) Electrochem Commun 4:305-309

17. Hellberg D, Scholz F, Schubert F, Lovric M, Omanovic D, Agmo Hernández V, Thede R (2005) J Phys Chem B 109:14715-14726

18. Stauffer V, Stoodley R, Agak JO, Bizzotto D (2001) J Electroanal Chem 516:73-82

19. Mårtensson C, Agmo Hernández V (2012) Ubiquinone-10 in gold-immobilized lipid membrane structures acts as a sensor for acetylcholine and other tetraalkylammonium cations.

Bioelectrochemistry. Doi: 10.1016/j.bioelechem.2012.03.009

20. Zutic V, Svetlicic V, Zimmermann AH, DeNardis NI, Frkanec R (2007) Langmuir 23:86478649

21. Agmo Hernández V, Scholz F (2006) Langmuir 22:10723-10731

22. Agmo Hernández V, Scholz F (2007) Langmuir 23:8650-8650

23. Milchev A (2008) Russ J Electrochem 44:619-645

24. Agmo Hernández V, Scholz F (2008) Bioelectrochemistry 74:149-156

25. Hermes M, Scholz F, Hardtner C, Walther R, Schild L, Wolke C, Lendeckel U (2011) Angew Chem Int Edit50:6872-6875

26. Hermes M, Czesnick C, Stremlau S, Stöhr C, Scholz F (2012) J Electroanal Chem 671:33-37 
27. Agmo Hernández V, Niessen J, Harnisch F, Block S, Greinacher A, Kroemer HK, Helm CA, Scholz F (2008) Bioelectrochemistry 74:210-216

28. Zander S, Hermes M, Scholz F, Groening A, Helm CA, Vollmer D, Lendeckel U, Schild L (2012) J Solid State Electr 16:2391-2397

29. Agmo Hernández V, Hermes M, Milchev A, Scholz F (2009) J Solid State Electr 13:639-649 30. Milchev A (1998) J Electroanal Chem 457:35-46

31. Milchev A (2002) Electrocrystallization. Fundamentals of Nucleation and Growth. Kluwer Academic Publishers, USA.

32. Agmo Hernández V, Milchev A, Scholz F (2009) J Solid State Electr 13:1111-1114

33. DeNardis NI, Zutic V, Svetlicic V, Frkanec R, Tomasic J (2007) Electroanal 19:2444-2450

34. Agmo Hernández V, Karlsson G, Edwards K (2011) Langmuir 27:4873-4883

35. Barbet J, Machy P, Truneh A, Leserman LD (1984) Biochim Biophys Acta 772:347-356

36. Szoka FC, Jacobson K, Papahadjopoulos D (1979) Biochim Biophys Acta 551:295-303

37. Straubinger RM, Hong K, Friend DS, Papahadjopoulos D (1983) Cell 32:1069-1079

38. Polyansky AA, Volynsky PE, Nolde DE, Arseniev AS, Efremov RG (2005) J Phys Chem B 109:15052-15059

39. Pyrkova DV, Tarasova NK, Pyrkov TV, Krylov NA, Efremov RG (2011) Soft Matter 7:25692579 


\section{Figure captions}

Fig. 1 Schematic 2-D representation of a liposome. An interior aqueous compartment is separated from the aqueous bulk solution by a lipid bilayer membrane. Lipids on each layer point their hydrophobic tails towards the inner part of the membrane, while the hydrophilic headgroups point towards the aqueous solutions

Fig. 2 a) Chronoamperometric trace obtained at $-0.7 \mathrm{~V}$ vs. $\mathrm{Ag} \mid \mathrm{AgCl}$ and $25^{\circ} \mathrm{C}$ in a suspension of $0.1 \mathrm{~g} \mathrm{~L}^{-1}$ of dimyristoyl phosphatidylcholine (DMPC) MLVs in $0.1 \mathrm{M} \mathrm{KCl}$. Each current spike arises from the adhesion and spreading of one MLV. The size of the peak is proportional to the size of the particular vesicle producing that signal. b) a magnification of the peak highligted in part (a). The shape of the peak (sharpness, time span/ height ratio, etc.) is related to the mechanical properties of the vesicle

Fig. 3 Number of adhesion spreading events $\mathrm{N}$ as a function of time determined experimentally (thick solid line) and calculated assuming diffusion control (dotted line), and a mixed mass transport/nucleation kinetics (dashed line, given by Eq. 1) control. Vertical bars represent the experimental standard error. Adapted from reference [29]

Fig. 4 Distribution of the probability $P_{\mathrm{m}}$ of obtaining $m$ adhesion-spreading signals after $100 \mathrm{~ms}$ at $-0.9 \mathrm{~V}$ vs. $\mathrm{Ag} \mid \mathrm{AgCl}$ (average = 2.4). The sample consists of DMPC GUVs $0.05 \mathrm{~g} \mathrm{~L}^{-1}$ in $0.1 \mathrm{M} \mathrm{KCl}$ at $25{ }^{\circ} \mathrm{C}$. The solid line represents the Poisson distribution calculated from the given average. Figure adapted from [32]

Fig. 5 Diagram illustrating the mechanism of fusion of DPPC liposomes with polystyrene (PS) nanoparticles. The darker spot in $\mathrm{L}_{\mathrm{a}}$ represents an active site. The formation of $\mathrm{N}$ and of $\mathrm{L}_{\mathrm{f}}$ was confirmed with Cryo-TEM and DLS experiments at different time points during the reaction (for details, see reference [34])

Fig. 6 Cartoon of the particle liposome clusters formed upon mixing of POPC liposomes in the liquid crystalline phase state and polystyrene nanoparticles (PS). Darker spots represent active sites in the lipid membrane. As several sites per liposome are active simultaneously, the liposome can attach to more than one particle at once. If a liposome is completely surrounded by particles (marked with a star in the figure), its contents cannot be released to the bulk solution, inhibiting the leakage response. DLS measurements confirmed the formation of large clusters in POPC:PS mixtures 
Fig. 1

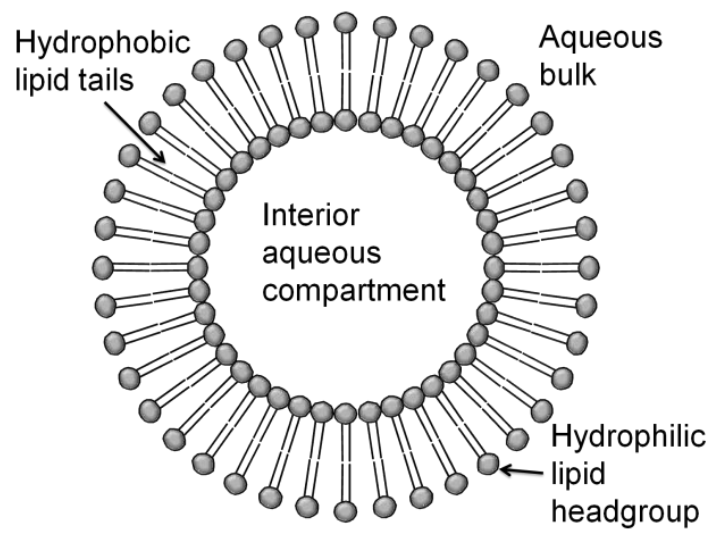


Fig. 2

a)

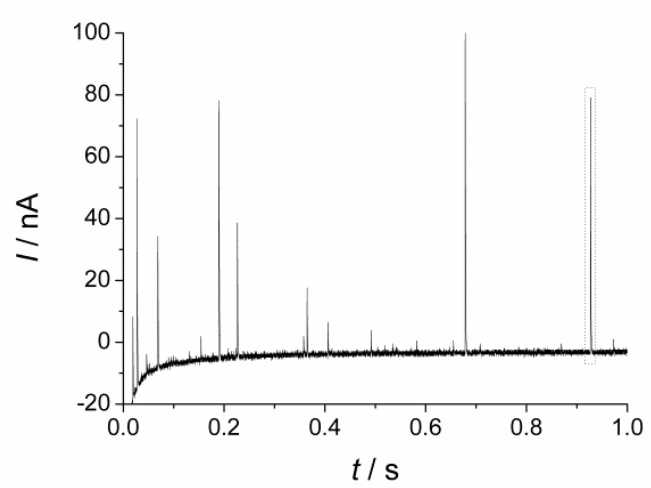

b)

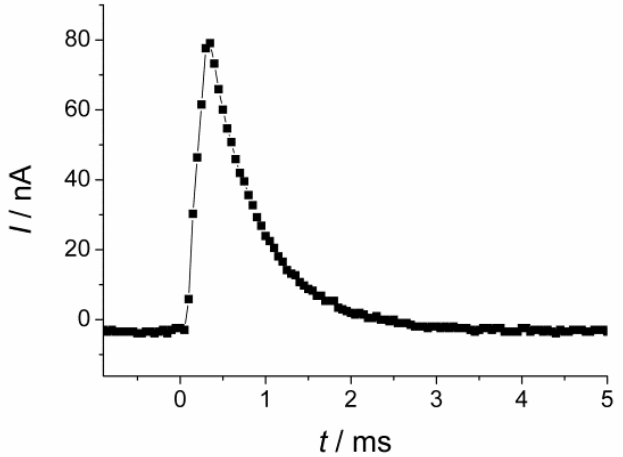


Fig. 3

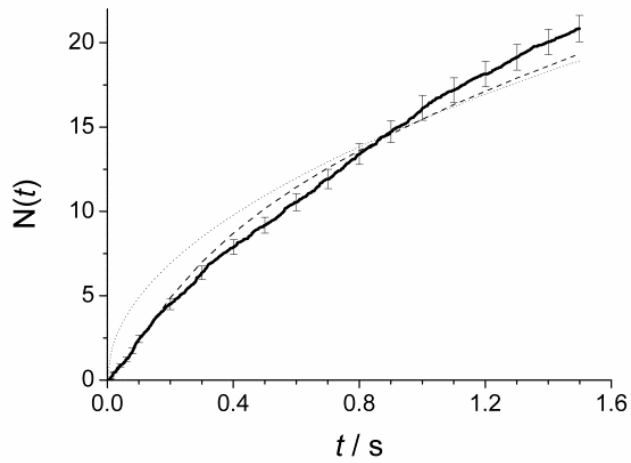


Fig. 4

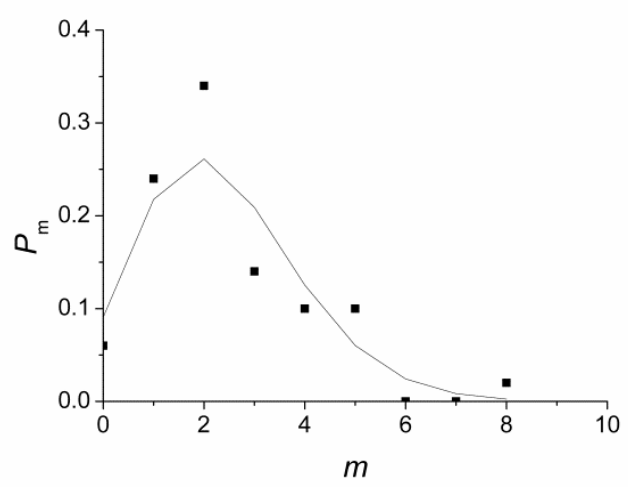


Fig. 5

a) Activation-deactivation

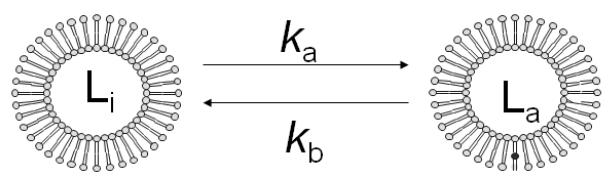

b) Adhesion ("nucleation")

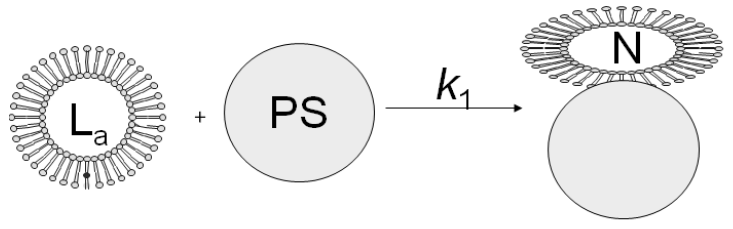

c)Rupture and spreading ("fusion")

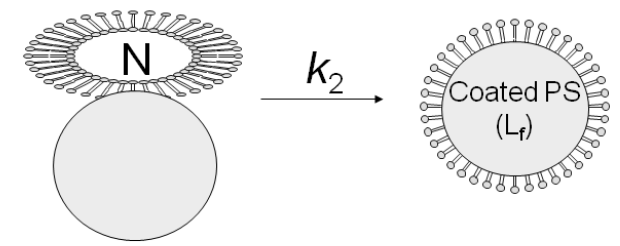


Fig. 6

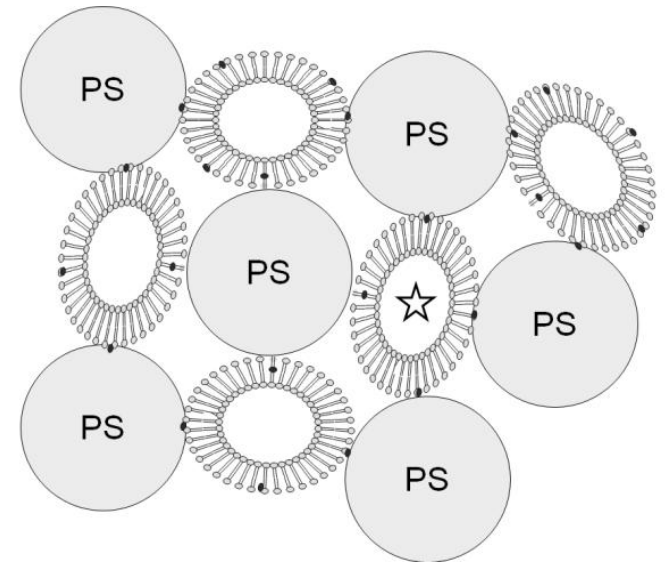

